\title{
Social awareness and price competition
}

\author{
Nada Ben Elhadj, ${ }^{*}$ Jean J. Gabszewicz ${ }^{\dagger}$ and Ornella Tarola ${ }^{\ddagger}$
}

\begin{abstract}
Human actions are often guided both by individual rationality and by social norms. In this paper we explore how market competition values the variants of a product, when these variants embody at different levels the requirements derived from some social norm. In a model where preferences of consumers depend partially on the levels of compliance of the variants with the social norm, we characterize the equilibrium path along which firms choose sequentially their level of compliance and their price.
\end{abstract}

Key words social norms, others-regarding preferences, vertical product differentiation

JEL classification D11, L13, Q58

Accepted 20 May 2014

\section{Introduction}

If a consumer buys a product which lacks any environment-friendly characteristics, he might have a bad conscience because environmental awareness is expected from him. His environmental attitude is influenced by friends, parents, partners, or by the media, but it is often not strong enough to push the market share of [environment-]incompatible products towards zero and that of the environmental-friendly substitute towards one. (Conrad 2005, p. 2)

Consider a consumer willing to buy some variant of a product. She has the choice between two variants, one at the exclusion of the other. The first variant, while perfectly satisfying her individualistic and private needs, lacks some environment-friendly characteristics. The alternative variant, by contrast, though less attractive from the consumer's private and individualistic viewpoint, far better meets the environmental requirements of the community in which this consumer lives. This would be the case, for instance, if the consumer, a fan of speed, has to buy a car and hesitates between very fast polluting car and one known for its green characteristics. Assuming that environmental awareness is very important in her community, this consumer might choose to buy green car, even if she privately prefers the fast car: doing otherwise would entail social disapproval so considerable that it would enforce a reversal of her private preferences between the two variants.

As an alternative example, consider a beverage containing fruit juice and some proportion of alcohol. According to the period and the national community under study, this proportion can vary between 0 and $100 \%$. For instance, during Prohibition it had to be equal to 0 , while in Western countries today there are no no explicit constraints. Nevertheless, for road safety reasons, most regions have adopted rules limiting the quantity of alcohol allowed in drivers' blood, with huge fines

\footnotetext{
ISG, University of Tunis, Le Bardo, Tunis, Tunisia.

CORE, Catholic University of Louvain, Louvain-La-Neuve, Belgium.

DISSE, University of Rome La Sapienza, Rome, Italy. Email: ornella.tarola@uniroma1.it
} 
to be paid when the edict is openly violated. These rules not only enhance the rise of an anti-alcohol social norm in the society, but also give rise to an objective punishment, should drivers not comply with the rule. A driver who likes alcohol might privately prefer to drink a variant of this beverage containing a higher proportion of alcohol than fruit juice. However, the fear of punishment might induce her to reverse her private preference, and rather consume a lighter drink with more fruit juice and less alcohol.

A final example is the attitude of tobacco consumers with respect to smoking. Thirty years ago, it was fashionable to smoke cigarettes since most movie stars were hard smokers, or at least behaved as if they were. Meanwhile, the disastrous effects of tobacco on health have been extensively documented and an anti-tobacco social norm has developed, leading for instance to the prohibition of smoking in public buildings, with the possibility of financial penalties for those breaking the rules.

All the above examples and comments reveal that, when making their shopping decisions, consumers are not only interested in meeting their private needs, but also guided in their choice by the degree to which it complies with some social norm. Furthermore, violation or weak compliance with the norm can generate a social punishment, while stronger compliance can produce a social reward. In the words of Elster (1989, p. 99), "for norms to be social, they must be shared by other people and partly sustained by their approval and disapproval. They are also sustained by the feelings of embarrassment, anxiety, guilt and shame that a person suffers at the prospect of violating them."

The duopoly theory developed hereafter is based on the judgment that, in some cases, human actions are typically guided both by individual rationality and social norms. Also it assumes that the variants of some good reflect more or less adequately the social driving force lying behind the norm. Consider a market with two different variants of a good embodying some characteristics complying more or less with a social norm, such as a beverage containing more or less alcohol, or a car in its green and brown variants. In this situation, the shopping decision cannot be viewed as if the level of compliance of the variants with the norm would not play a role in the choice of the consumer: it would deny the right of the consumer to be guided by the importance she pays to herothers-regarding preferences in her choice of appliance. Accordingly, we propose in the following to incorporate in the utility function of the consumer a new term depending, for each variant, on the level of its compliance with the social norm. Furthermore, we introduce the idea of "social reward" and "punishment" by assuming that the utility for a specific variant does not depend only on its own level of compliance with the norm, but also on the social reward-punishment differential: the larger this differential, the higher the difference between the reward when buying the most compliant variant and the punishment when buying the other one variant. This assumption naturally flows from the fact that consumers examine the relative compliance of a variant with the norm along a compliance ladder, as this position contributes to defining their own relative position on the social ladder: the higher the variant's compliance with the norm, the higher the social position of its buyer in the social community. Incorporating these new ingredients into the model of course affects the demand functions of firms, whatever the level of compliance of their variants with the social norm. Finally, we assume that firms are able to select this level of compliance in some domain. The decisions about prices and variants are taken sequentially, first the level of compliance and then price, and we examine the properties of the subgame perfect equilibrium of this sequential game.

Our findings are as follows. According to the relative importance consumers assign to the compliance of the variants with the social norm, either both firms are active in the market at positive equilibrium prices, or only the one with the highest level of compliance is active, the rival firm being excluded from the market. The smaller the social reward-punishment differential between the variants, the higher the chance of observing two variants at equilibrium. In contrast, when this differential is large, only the variant with the higher level of compliance can survive at equilibrium. 
In this case, two subcases must be distinguished. In the first, the owner of this variant behaves as a monopolist, but must set a price sufficiently low to keep the rival firm out of the market. In the second, when the social reward-punishment differential is sufficiently large, the rival can no longer pose a threat to the monopolist. Then, it is optimal for the monopolist to quote the pure monopoly price. Finally, the levels of compliance are fully determined in the first stage of the game. When both firms are active at equilibrium prices corresponding to the top level of compliance, the firm with the higher level of compliance chooses precisely this top level while the firm with the low level of compliance chooses a strictly smaller level of compliance than the top one. Nevertheless, the higher the threat of punishment, the more compliant the choice of the latter.

The model explored in this paper shares several features with the traditional vertical product differentiation model (Gabszewicz and Thisse 1979). Nevertheless, the introduction of others-regarding preferences, via the existence of a social norm, considerably alters the predictions of the latter. The differences and similarities between the two models and their predictions will be systematically pointed out along the equilibrium analysis.

A striking example of goods subject to the existence of a social norm are environmental products. It is not surprising that applying our results to environmental economics leads to interesting insights in this specific field. Moreover, as will be shown later, our approach displays some properties directly comparable with those emerging in models on the end-of-pipe approach. ${ }^{1}$ Thus, our paper opens the door to a clear comparison between measures based on green consumerism and the traditional environmental regulation.

In the next section we present the formal model. Then we proceed with the equilibrium analysis in Section 3. We conclude with a short conclusion.

\section{The model}

Consider two variants of the same product, $A$ and $B$, and some social norm developed in a community whose members are identified by the interval $[0, \bar{\mu}], 0<\bar{\mu}$. We say that variant $A$ fits the social norm better than variant $B$ if everybody in the community feels that consuming variant $A$ complies better with the norm than variant $B$. Thus, assume that variant $A$ fits the social norm better than variant $B$. Firm $A$ (firm $B$ ) produces variant $A$ (variant $B$ ) at no cost. ${ }^{2}$

Let $\mu v_{A}\left(\mu v_{B}\right)$ with $\mu \in[0, \bar{\mu}]$ be a component of the satisfaction - we call it individual $\mu$ 's social concern for product $A(B)$ - obtained by consumer $\mu$ when consuming one unit of variant $A(B)$; $v_{A}\left(v_{B}\right)$ represents for all consumers the rate at which variant $A(B)$ fits the social norm, or its level of compliance, so that $v_{A}>v_{B}$. All consumers agree on the levels of compliance $v_{A}$ and $v_{B}$, but we assume them to be ranked in the domain $[0, \bar{\mu}]$ according to the size of their social awareness. This introduces heterogeneity in the population since all individuals are not uniformly concerned with the social norm: the closer to 0 , the weaker the social awareness of individual $\mu$ in the community.

Furthermore, we assume that consuming variant $A$ rather than variant $B$ also affects the satisfaction of the consumer because consuming a variant better complying with the social norm generates

1 Typically, the end-of pipe technologies allow pollution emissions to be reduced by implementing add-on measures rather than using cleaner inputs or production process (Copeland and Taylor 1994).

2 This assumption is a little awkward because one can imagine that a more compliant variant should entail some costly investments not obligatory when the compliance of the variant is a less important variable. However, if these investments' costs are convex in quality and fixed in quantity, they would not alter the following analysis, while reducing at equilibrium the optimal level of compliance of the variant $v_{A}$. This assumption is introduced for simplicity. 
a socially worthy identity, transformed in turn into a social reward. ${ }^{3}$ Conversely, consuming a variant less complying with the social norm generates a feeling of social disapproval transformed in turn into a social punishment. ${ }^{4}$ An immediate byproduct of the above is that the benefit from consuming variant $A$ rather than variant $B$ is higher, the larger the difference between $v_{A}$ and $v_{B}$. In the following, we call this difference the reward-punishment differential. Thus, we add to the utility $\mu v_{A}$ another term measuring the social benefit (reward) of consuming one unit of variant $A$ rather than variant $B$, namely $\alpha\left(v_{A}-v_{B}\right)$. Symmetrically, we add to the utility $\mu v_{B}$ the term $\alpha\left(v_{B}-v_{A}\right)$ to capture the frustration (punishment) incurred by the consumer when she consumes one unit of variant $B$ rather than $A$. Finally, the utility for consumer $\mu$ when choosing variant $A$ is given by

$$
V_{A}(\mu)= \begin{cases}\mu v_{A}+\alpha\left(v_{A}-v_{B}\right) & \text { if she chooses variant } A, \\ 0 & \text { otherwise, }\end{cases}
$$

and when choosing variant $B$ by

$$
V_{B}(\mu)= \begin{cases}\mu v_{B}+\alpha\left(v_{B}-v_{A}\right) & \text { if she chooses variant } B, \\ 0 & \text { otherwise. }\end{cases}
$$

It is assumed that

$$
0<\alpha<\bar{\mu}
$$

with $\alpha \in] 0,1]$.

When there exist markets in which the two variants can be exchanged against money, at unit prices $p_{i}, i=A, B$, the utility after purchasing variant $A(B)$ is given by a quasi-linear utility function $U_{A}(\mu)=V_{A}(\mu)-p_{A}\left(U_{B}(\mu)=V_{B}(\mu)-p_{B}\right)$.

Finally we assume that the level of compliance is chosen by the firms in some domain $[\underline{v}, \bar{v}]$ : there exists a lowest level of compliance $\underline{v}$ such that, if the proposed variant did not meet this level, the social norm would be violated. In contrast, there exists a highest level of compliance $\bar{v}$ guaranteeing that the social norm is fully satisfied.

\section{Equilibrium analysis}

We consider a two-stage game. In the first stage, firms have to decide the extent to which their variant complies with the social norm, namely, $v_{i}, i=A, B$. In the second stage of the game, each firm chooses its price $p_{i}, i=A, B$.

The game is solved by backward induction. Assuming that firms choose first the level of compliance $v_{i}, i=A, B$, in the domain $[\underline{v}, \bar{v}]$, and then their price, we determine first the demand for each firm as a function of $p_{i}$ and $v_{i}$, for $i=A, B$. Then we determine the price equilibrium for given levels of compliance, and, finally, we identify the optimal level of compliance.

Traditionally, when deciding whether to purchase a good, a consumer takes into account the benefit deriving from the intrinsic characteristics of the good and the price at which it is sold. As we have just seen, the existence of a social norm introduces further ingredients into her decision-making process. In particular, when an individual takes into consideration the consequences deriving from a specific purchase in terms of social reward and punishment, a variant which would not be a priori

3 This social reward can sometimes assume the shape of a subsidy, as in the example of the solar panels above.

4 This social punishment may sometimes assume the shape of a tax or a fine, like in the example of the car drivers above. 
considered in the shopping list can be bought for its social value. In order to embed these ingredients in our formal analysis, we define both the marginal consumer $\hat{\mu}\left(p_{A}, p_{B}\right)$ indifferent between buying variant $B$ and variant $A$ at prices $p_{A}$ and $p_{B}$ (solution of the equation $\left.V_{A}(\mu)-p_{A}=V_{B}(\mu)-p_{B}\right)$, namely,:

$$
\hat{\mu}\left(p_{A}, p_{B}\right)=\frac{p_{A}-p_{B}}{v_{A}-v_{B}}-2 \alpha,
$$

and the consumer $\mu_{i}\left(p_{i}\right)$ who is indifferent between buying product $i$ and not buying at all (solution of the equation $\left.U_{i}\left(\mu_{i}, p_{i}\right)=0\right)$. Accordingly, we write

$$
\mu_{B}\left(p_{B}\right)=\frac{p_{B}+\alpha\left(v_{A}-v_{B}\right)}{v_{B}}(>0)
$$

and, in the same way,

$$
\mu_{A}\left(p_{A}\right)=\frac{p_{A}-\alpha\left(v_{A}-v_{B}\right)}{v_{A}}>0 \text { if } p_{A}>\alpha\left(v_{A}-v_{B}\right) .
$$

From the above definitions, we provide in Appendix I the demand functions faced by firm $B$ and firm $A$, respectively, namely,

$$
D_{B}= \begin{cases}\bar{\mu}-\mu_{B}\left(p_{B}\right) & \text { iff } \mu_{B}\left(p_{B}\right)<\bar{\mu}<\hat{\mu}\left(p_{A}, p_{B}\right) \\ \hat{\mu}\left(p_{A}, p_{B}\right)-\mu_{B}\left(p_{B}\right) & \text { iff } \mu_{B}\left(p_{B}\right) \leq \hat{\mu}\left(p_{A}, p_{B}\right) \leq \bar{\mu} \\ 0 & \text { iff } \hat{\mu}\left(p_{A}, p_{B}\right)<\mu_{A}\left(p_{A}, p_{B}\right)<\bar{\mu}\end{cases}
$$

and

$$
D_{A}= \begin{cases}\bar{\mu}-\mu_{A}\left(p_{A}, p_{B}\right) & \text { iff } \hat{\mu}\left(p_{A}, p_{B}\right)<\mu_{A}\left(p_{A}, p_{B}\right)<\bar{\mu} \\ \bar{\mu}-\hat{\mu}\left(p_{A}, p_{B}\right) & \text { iff } \mu_{B}\left(p_{A}, p_{B}\right) \leq \hat{\mu}\left(p_{A}, p_{B}\right) \leq \bar{\mu} \\ 0 & \text { iff } \mu_{B}\left(p_{A}, p_{B}\right)<\bar{\mu}<\hat{\mu}\left(p_{A}, p_{B}\right) .\end{cases}
$$

Notice that, in the case $\mu_{B}\left(p_{A}, p_{B}\right)<\bar{\mu}<\hat{\mu}\left(p_{A}, p_{B}\right)$, the demand function for firm $B$ turns out to be $\bar{\mu}-\mu_{B}\left(p_{A}, p_{B}\right)>0$, while that for firm $A$ is zero. Thus, it is immediately concluded that in this range of $\mu$-parameters, firm $B$ monopolizes the market, while firm $A$ is inactive. By the same token, firm $A$ monopolizes the market whenever $\hat{\mu}\left(p_{A}, p_{B}\right)<\mu_{A}\left(p_{A}, p_{B}\right)<\bar{\mu}$, firm $B$ being inactive in this range of $\mu$-parameters. Finally, in the case where $\mu_{B}\left(p_{A}, p_{B}\right) \leq \hat{\mu}\left(p_{A}, p_{B}\right) \leq \bar{\mu}$, firm $A$ and firm $B$ share the market.

We can now start solving the game. To this end, we move to the analysis of the second stage where price competition between firms takes place, assuming that the level of compliance has been chosen by each firm at the first stage.

\subsection{The second stage game: choosing the price}

Let $\Pi_{i}$ be the profit function of firm $i, i=A, B$, defined by

$$
\Pi_{i}\left(v_{i}, p_{i}, v_{j}, p_{j}\right)=p_{i} D_{i}\left(p_{i}, p_{j}\right) .
$$

In order to identify the equilibrium prices, we first define the best reply functions ${ }^{5}$ of firm $A$ and firm $B$. Notice that in order to solve this stage of the game, the level of compliance is assumed to be exogenous so that we can solve for the equilibrium prices with respect to $v_{A}$ and $v_{B}$.

\footnotetext{
5 Details on these functions are provided in Appendix II.
} 
We find that the prices observed at equilibrium crucially depend on the ratio $v_{A} / v_{B}$. Depending on whether the value of this ratio is smaller or larger than $H_{1}$, with $H_{1}=\bar{\mu} / 2 \alpha$, the corresponding equilibrium prices lead to two different market structures. ${ }^{6}$ In the first, observed when $v_{A} / v_{B} \leq H_{1}$, both firms are active in the market and equilibrium prices $p_{A}^{*}$ and $p_{B}^{*}$ are positive and given by

$$
\begin{aligned}
p_{A}^{*} & =2 \bar{\mu} v_{A} \frac{v_{A}-v_{B}}{4 v_{A}-v_{B}}\left(3 \alpha v_{A}-\alpha v_{B}\right), \\
p_{B}^{*} & =\left(v_{A}-v_{B}\right) \frac{\bar{\mu} v_{B}-2 \alpha v_{A}}{4 v_{A}-v_{B}} .
\end{aligned}
$$

Notice that the condition $v_{A} / v_{B} \leq H_{1}$ cannot be met without the inequality $H_{1}>1$ being met, implying $\bar{\mu}>2 \alpha$ from the very definition of $H_{1}$. The larger the value of $\bar{\mu}$, the more significant the heterogeneity among consumers and the larger the domain of $\alpha$-values satisfying the condition $\bar{\mu}>2 \alpha$. Thus, the market can sustain both variants if the heterogeneity among consumers $(\bar{\mu})$ is sufficiently large and/or the role of punishment and reward in consumers' decisions is not too significant.

The equilibrium prices identified above are exactly those which would obtain in a vertical product differentiation model if the reward-punishment differential did not play any role. Interpreting the reward-punishment differential as a difference between a subsidy and a tax rate on the two variants, we see that the existence of this subsidy and tax increases (decreases) the equilibrium price $p_{A}^{*}\left(p_{B}^{*}\right)$ compared with the equilibrium values observed in the traditional vertical differentiation model with an uncovered market. The rationale behind this finding is that the reward-punishment differential strengthens the traditional market power of firm $A$, while weakening that of its rival firm $B$.

In contrast to the above analysis, firm $A$ evicts firm $B$ from the market in the case where $\frac{v_{A}}{l} v_{B}>H_{1}$. Indeed, variant $A$ is so significantly compliant with the consumption norm (compared with variant $B$ ) that no consumer is willing to buy variant $B$, whatever its price. In this latter case, the equilibrium prices of firms $A$ and $B$ are given, respectively, by

$$
p_{A}^{+}=\frac{\left(v_{A}-v_{B}\right)\left(\alpha v_{A}+\alpha v_{B}\right)}{v_{B}}, p_{B}^{+}=0, \quad \text { if } \frac{v_{A}}{v_{B}} \leq H_{2},
$$

and

$$
p_{A}^{\circ}=\frac{1}{2}\left(\bar{\mu} v_{A}+\alpha\left(v_{A}-v_{B}\right)\right), p_{B}^{\circ}=[0, y], \quad \text { if } \frac{v_{A}}{v_{B}}>H_{2},
$$

with $H_{2}=\frac{1}{4 \alpha}\left(\alpha+\bar{\mu}+\sqrt{2 \alpha \bar{\mu}+9 \alpha^{2}+\mu^{2}}\right)$.

Observe that, when $v_{A} / v_{B} \leq H_{2}$, the equilibrium price $p_{A}^{+}$coincides with the so-called "limit price" arising in a vertical product differentiation model: this price, with $p_{A}^{+}<p_{A}^{\circ}$, is set sufficiently low to keep the rival $B$ out of the market at equilibrium. ${ }^{7}$ In this case, the equilibrium price $p_{A}^{+}$turns out to be higher, the higher $\alpha$. Indeed, a high value of $\alpha$ magnifies the role of social punishment, thereby reducing ceteris paribus the benefit deriving from purchasing variant $B$. As a consequence, firm $A$ can keep its competitor out of the market at a relatively higher equilibrium price $p_{A}^{+}$, compared with a scenario with a weaker punishment incurred by consumers when buying variant $B$. Accordingly, in this scenario where $v_{A} / v_{B} \leq H_{2}$ holds, firm $B$, in spite of being inactive in the

6 See Appendix III for details.

7 Notice, however, that, contrary to the limit pricing strategy used by the high-quality firm in a vertically differentiated market, the limit price strategy used here by firm $A$ does not imply that the market is covered. 
market, still affects the equilibrium outcome. In contrast, when $v_{A} / v_{B}>H_{2}$, the monopolist $A$ is a "true" monopolist to the extent that its rival can no longer affect the equilibrium. By the way, it is easy to check that the price $p_{A}$ is the pure monopoly price corresponding to the situation where firm $B$ would simply not exist. In this case, ${ }^{8}$ one can see that the resulting market share $D_{A}=\left(\bar{\mu} v_{A}+\alpha\left(v_{A}-v_{B}\right)\right) / 2 v_{A}$, when assuming $\alpha=0$, coincides with that observed in the "true" monopoly case.

We can summarize the above findings as follows.

Proposition 1 At the subgame price equilibrium, depending on the value of the ratio $v_{A} / v_{B}$, either both firms are active in the market at positive equilibrium prices $\left(v_{A} / v_{B} \leq H_{1}\right)$, or only the firm endowed with the higher level of compliance is active, the rival firm being excluded $\left(v_{A} / v_{B}>H_{1}\right)$.

Further, we have the following corollary:

Corollary 1 When the market is monopolized by firm A, its optimal price is given by $\min \left[p_{A}^{+}, p_{A}\right]$.

\subsection{The first stage game: choosing the level of compliance}

Let us now consider the first stage of the game when the firms choose their level of compliance $v_{i}$, in the domain $[\underline{v}, \bar{v}], i=A, B$. First, we remark that the market can sustain both variants if the heterogeneity among consumers $(\bar{\mu})$ is sufficiently large and/or the role of punishment and reward in consumers' decisions is not too significant, namely $H_{1} \geq 1$. Otherwise, when $H_{1}<1$, only firm $A$ can be active in the market, firm $B$ being evicted.

Notice that, in both these subdomains, the following lemma holds:

Lemma 1 The profit function of firm $A$ is monotonically increasing in $v_{A}$ for all $v_{B} \in[\underline{v}, \bar{v}]$. Thus, its best reply function is always given by $\varphi_{A}\left(v_{B}\right)=\bar{v}$.

Regarding firm $B$, in the domain where it is active, namely $H_{1} \geq 1$, its best reply function $v_{B}\left(v_{A}\right)$ is given by

$$
v_{B}\left(v_{A}\right)=\gamma v_{A}, \quad \gamma=\frac{2 \bar{\mu}-3 \alpha+\sqrt{(2 \mu-\alpha)(23 \alpha+2 \mu)}}{7 \mu-4 \alpha}<1 .
$$

Thus, the following proposition holds:

Proposition 2 When $\bar{\mu} / 2 \alpha<1$, firm $B$ is inactive, and firm A selects $\bar{v}$. Otherwise, with both firms being active in the market, the levels of compliance corresponding to the Nash equilibrium are given by $(\bar{v}, \gamma \bar{v})$.

It is worth noticing that this result is not in line with the traditional findings in vertical differentiation. Indeed, in vertical differentiation models, the low-quality firm can be inactive under particular conditions on the model parameters only if the market is covered, with all consumers buying the high-quality variant. Then a finiteness property holds, according to which only a single firm can survive at equilibrium (see Gabszewicz and Thisse 1980); when this is the case, the market

8 Notice that market share corresponding to the monopoly case when a limit price is quoted is defined as $D_{A}^{+}=$ $\left(\bar{\mu} v_{B}-\alpha\left(v_{A}-v_{B}\right)\right) / v_{B}$. 
is a natural monopoly. In contrast, when the market is uncovered, the finiteness property does not apply.

In the current model, by contrast, there exist some circumstances under which firm $B$ cannot stay active in the market due to the existence of social norms, regardless of the coverage of the market at equilibrium. In particular, it follows from the above that when $\bar{\mu} / 2 \alpha<1$, the firm $B$ with the lower level of compliance is kept out of the market. Also, this finding holds whatever the level of market coverage as it depends only on the intensity of social norms $\alpha$ with respect to the consumers' heterogeneity in the market $\bar{\mu}$.

Finally, evaluating the derivative $\partial v_{B} / \partial \alpha$ in the subdomain defined by the condition $1 \leq H_{1}$ (see Appendix IV), we get the following proposition:

Proposition 3 At the subgame perfect Nash equilibrium (SPNE), the compliance level of firm $B$ in the range of parameters where it can be active in the market increases with $\alpha$.

Accordingly, the parameter $\alpha$ can be viewed as an incentive for the less complying firm $B$ to increase its level of compliance. When $\alpha$ increases, the punishment for deviating from the social norm becomes more and more influential on the consumer's decision. At some point, when $\alpha$ becomes so high that the condition $\bar{\mu}>2 \alpha$ is violated (thereby implying that the inequality $v_{A} / v_{B} \leq H_{1}$ no longer holds), the less complying firm is driven out of the market: all consumers feel that it sells a variant insufficiently complying with the social norm. It is easy to see that, when $\alpha=0$, the model considered above boils down to the usual model of vertical product differentiation studied by Gabszewicz and Thisse (1979).

\subsection{A natural extension}

One may wonder to what extent this interdependence among preferences plays a key role in the analysis. For example, is the SPNE robust to the idea that consuming a variant less compliant with the social norm does not generate a feeling of social disapproval transformed in turn into a social punishment? This question seems reasonable, mainly when thinking of communities where social awareness is weak. Casual observations show that social attitudes are not uniform worldwide and can increase with education, thereby being particularly strong among high-skilled and high-income people. In these circumstances, our assumption regarding the positive role of social punishment turns out to be questionable. In order to analyze this issue, one needs to perform the analysis under the assumption that $V_{B}(\mu)=\mu v_{B}$. This allows us to capture the idea that some consumers do not care about the existence of goods complying with the social norm when evaluating benefits from consumption. While knowing that there exist variants with different degrees of compliance, these consumers do not feel embarrassment or guilt when buying the variant $v_{B}$.

Rather interestingly, it emerges that in this circumstance, the unique price equilibrium is given by

$$
\begin{aligned}
& \check{p}_{A}=\left(v_{A}-v_{B}\right) \frac{2 \bar{\mu} v_{A}+2 \alpha v_{A}-\alpha v_{B}}{4 v_{A}-v_{B}}, \\
& \check{p}_{B}=v_{B}\left(v_{A}-v_{B}\right) \frac{\bar{\mu}-\alpha}{4 v_{A}-v_{B}}
\end{aligned}
$$


such that both firms are active in the market achieving the following profits:

$$
\begin{aligned}
\check{\Pi}_{L} & =\frac{v_{B}\left(v_{A}-v_{B}\right)(\bar{\mu}-\alpha)^{2} v_{A}}{\left(4 v_{A}-v_{B}\right)^{2}}, \\
\check{\Pi}_{H} & =\frac{\left(v_{A}-v_{B}\right)\left(2 \bar{\mu} v_{A}+2 \alpha v_{A}-\alpha v_{B}\right)^{2}}{\left(4 v_{A}-v_{B}\right)^{2}} .
\end{aligned}
$$

Furthermore,

$$
\frac{\partial}{\partial v_{A}} \check{\Pi}_{H}>0
$$

and the optimal level of compliance for the less compliance variant $\check{v}_{B}$ is given by $\breve{v}_{B}=\frac{4}{7} v_{A}$. It is worth noting that this level of compliance coincides with that which would be observed in the traditional model of vertical differentiation with partial coverage of the market. Moreover, at this SPNE both firms are always active in the market.

Thus, whenever social awareness occurs only among people buying the more compliant good, the role of others-regarding preferences in defining market equilibrium turns out to be drastically less significant compared to the alternative case where social rewards and social punishment affect consumption.

\section{An application to environmental economics}

As stated in Section 1, environmental economics constitutes a natural field for applying the above results. In this interpretation, the variant with the higher degree of compliance should be viewed as a "green" product, the "brown" one being identified with the firm showing the lowest level of compliance with the environmental norm. In this strand of the literature, the core question is to identify means for reducing pollution damage at the international level. In Europe, the environmental policy is identified by the EU Emission Trading Scheme. Launched in 2005, this scheme is mainly based on a "cap and trade" principle: a cap defines the total amount of greenhouse gases that can be emitted by producers. Nevertheless, this approach has been questioned, as unilateral environmental policies can drive out firms to countries with less stringent regulation (the so-called "pollution havens"), or incite these countries to use environmental dumping to attract foreign direct investment.

Our analysis reveals that there exist alternative ways, based on profit-driven mechanisms, to sustain green production, with positive effects on pollution abatement. Assuming that the pollution damage decreases with the level of compliance, our results can be interpreted as follows. First, whatever the equilibrium market configuration, pollution abatement is driven by the existence of others-regarding preferences, compared with the situation where consumers' behavior would be only shaped by individual rationality. In the case when both firms are active at equilibrium, the compliance level selected by both firms is higher than it would be without such preferences, while the market share of the brown variant shrinks. As for the monopoly case, the pollution abatement follows directly from the fact that the brown variant must simply disappear from the market.

Furthermore, the above pollution abatement is a direct consequence of the market mechanism, rather than following from an explicit environmental policy. Thanks to this, it escapes the carbon leakage phenomenon that occurs when an explicit unilateral abatement policy is introduced, inducing polluting firms to relocate to pollution havens with less stringent regulation (see Sanna-Randaccio 
and Sestini 2012). Indeed, the brown firm of our model is prevented from relocating to a pollution haven while selling its variant in the home market: no consumer would be interested in buying this variant due to its insufficient level of compliance with the social norm.

\section{Conclusion}

In this paper we explore how duopoly market competition values the variants of a product, when these variants embody at different levels the requirements derived from some social norm. Introducing a model in which preferences of consumers depend partially on the levels of compliance of the variants with the social norm, we characterize the equilibrium path along which firms choose sequentially their level of compliance and their price. We conclude that the dependence of preferences on the level of compliance exerts a beneficial effect on the choice of variants by firms. In particular, when interpreting these results in the framework of environmental concern, they reveal the existence of a pollution abatement obtained independently from any explicit environmental policy.

While economists generally adopt a methodological position of strict individualism when depicting consumers' behavior, one must recognize that consumers' decisions are often significantly influenced by their social environment and the set of values that it conveys. Of course this recognition does not simplify the analysis of market behavior because it introduces externalities in the preferences of individuals. Nevertheless, it seems useless to get around this difficulty: more and more frequently, consumption decisions involve elements borrowed from the social sphere of individuals. Think of tobacco, drugs, brown products, arms and other goods, viewed as nuisances by a majority of the community in which consumers are immersed. The present paper is an essay to explore this world using the traditional tools of economic analysis.

\section{Appendix I}

\section{Demand functions}

First, notice that $\hat{\mu}-\mu_{B}$ and $\hat{\mu}-\mu_{A}$ have the same sign as $\mu_{A}-\mu_{B}$.

Let us denote by $\bar{p}_{B}=\bar{\mu} v_{B}-\alpha\left(v_{A}-v_{B}\right)$ the reservation price of variant $B$ for the consumer $\bar{\mu}$ such that for any $p_{B}>\bar{p}_{B}$, no consumer is willing to buy variant $B$. Then, let us denote by $p_{B}^{B}=p_{A}-(\bar{\mu}+2 \alpha)\left(v_{A}-v_{B}\right)$, for given $p_{A}$, the value of price $p_{B}$ corresponding to which consumer $\bar{\mu}$ is indifferent between buying variant $A$ and buying variant $B$. Finally, let us denote by $\breve{p}_{B}=\frac{v_{B}}{v_{A}} p_{A}-\left(v_{A}-v_{B}\right) \frac{\alpha v_{A}+\alpha v_{B}}{v_{A}}$ the value of price $p_{B}$ such that, for any $p_{B} \leq \breve{p}_{B}, \hat{\mu} \leq \bar{\mu}$. Symmetrically, we denote by $\bar{p}_{A}=\bar{\mu} v_{A}+\alpha\left(v_{A}-v_{B}\right)$ the price $p_{A}$ such that no consumer is willing to buy variant $A$ for $p_{A}>\bar{p}_{A}$, and $\breve{p}_{A}=\frac{v_{A}}{v_{B}} p_{B}+\left(v_{A}-v_{B}\right) \frac{\alpha v_{A}+\alpha v_{B}}{v_{B}}$ the value of price $p_{A}$ such that, for any $p_{A} \leq \breve{p}_{A}, \mu_{B} \leq \hat{\mu} \leq \bar{\mu}$. Finally, let $p_{A}^{B}=(\bar{\mu}+2 \alpha)\left(v_{A}-v_{B}\right)+p_{B}$ be the value of $p_{A}$ corresponding to which $\bar{\mu}$ is indifferent between buying variant $A$ and variant $B$, given $p_{B}$.

Thus, we can split each demand function into two separate functions as follows. If $p_{A} \geq \bar{\mu} v_{A}+\alpha\left(v_{A}-v_{B}\right)$, then firm $B$ 's demand function is given by

$$
D_{B}= \begin{cases}\bar{\mu}-\frac{p_{B}+\alpha\left(v_{A}-v_{B}\right)}{v_{B}} & \text { if } p_{B} \leq \bar{p}_{B}, \\ 0 & \text { if } p_{B} \geq \bar{p}_{B},\end{cases}
$$

while if $p_{A} \leq \bar{\mu} v_{A}+\alpha\left(v_{A}-v_{B}\right)$, then

$$
D_{B}= \begin{cases}\bar{\mu}-\frac{p_{B}+\alpha\left(v_{A}-v_{B}\right)}{v_{B}} & \text { if } p_{B} \leq p_{B}^{B}, \\ \frac{p_{A}-p_{B}}{v_{A}-v_{B}}-2 \alpha-\frac{p_{B}+\alpha\left(v_{A}-v_{B}\right)}{v_{B}} & \text { if } p_{B}^{B} \leq p_{B} \leq \breve{p}_{B}, \\ 0 & \text { if } p_{B} \geq \breve{p}_{B} .\end{cases}
$$


Furthermore,

$$
\min \left\{\bar{p}_{B}, p_{B}^{B}\right\}= \begin{cases}\bar{p}_{B} & \text { if } \mu_{A} \geq \bar{\mu} \\ p_{B}^{B} & \text { if } \mu_{A} \leq \bar{\mu} .\end{cases}
$$

Indeed, the inequality $\bar{p}_{B} \leq p_{B}^{B}$ implies that $p_{A} \geq \bar{\mu} v_{A}+\alpha\left(v_{A}-v_{B}\right)$ or $\bar{\mu} \leq \mu_{A}$, so that $D_{A}=0$. Thus, firm $B$ may obtain the whole demand of the market or be inactive depending on the position of $\mu_{B}$ with respect to $\bar{\mu}$. In particular, $D_{B}=\bar{\mu}-\mu_{B}$ if $\mu_{B}<\bar{\mu}$, while $D_{B}=0$ if $\mu_{B} \geq \bar{\mu}$.

Tuning to firm $A$ 's demand function, if $p_{B} \geq \bar{\mu} v_{B}-\alpha\left(v_{A}-v_{B}\right)$, then it is given by

$$
D_{A}= \begin{cases}\bar{\mu}-\frac{p_{A}-\alpha\left(v_{A}-v_{B}\right)}{v_{A}} & \text { if } p_{A} \leq \bar{p}_{A}, \\ 0 & \text { if } p_{A} \geq \bar{p}_{A},\end{cases}
$$

while if $p_{B} \leq \bar{\mu} v_{B}-\alpha\left(v_{A}-v_{B}\right)$, then

$$
D_{A}= \begin{cases}\bar{\mu}-\frac{p_{A}-\alpha\left(v_{A}-v_{B}\right)}{v_{A}} & \text { if } p_{A} \leq \breve{p}_{A}, \\ \bar{\mu}-\left(\frac{p_{A}-p_{B}}{v_{A}-v_{B}}-2 \alpha\right) & \text { if } \breve{p}_{A} \leq p_{A} \leq p_{A}^{B}, \\ 0 & \text { if } p_{A} \geq p_{A}^{B}\end{cases}
$$

Given that,

$$
\min \left\{\bar{p}_{A}, \breve{p}_{A}\right\}= \begin{cases}\bar{\mu} v_{A}+\alpha\left(v_{A}-v_{B}\right) & \text { if } \mu_{B} \geq \bar{\mu}, \\ \frac{v_{A}}{v_{B}} p_{B}+\left(v_{A}-v_{B}\right) \frac{\alpha v_{A}+\alpha v_{B}}{v_{B}} & \text { if } \mu_{B} \leq \bar{\mu},\end{cases}
$$

the inequality $\breve{p}_{A} \geq \bar{p}_{A}$ implies $p_{B} \geq \bar{\mu} v_{B}-\alpha\left(v_{A}-v_{B}\right)$ or $\bar{\mu} \leq \frac{p_{B}}{v_{B}}+\frac{\alpha\left(v_{A}-v_{B}\right)}{v_{B}}=\mu_{B}$, so that $D_{B}=0$. Thus firm $A$ obtains the whole demand of the market or is inactive depending on the position of $\mu_{A}$ with respect to $\bar{\mu}$. In particular, $D_{A}=\bar{\mu}-\mu_{A}$ if $\mu_{A}<\bar{\mu}$, while $D_{A}=0$ if $\mu_{A} \geq \bar{\mu}$.

Summarizing the above findings allows us to write the following demand functions:

$$
D_{B}= \begin{cases}\bar{\mu}-\frac{p_{B}+\alpha\left(v_{A}-v_{B}\right)}{v_{B}} & \text { if } p_{B} \leq \min \left\{\bar{p}_{B}, p_{B}^{B}\right\}, \\ \frac{p_{A}-p_{B}}{v_{A}-v_{B}}-2 \alpha-\frac{p_{B}+\alpha\left(v_{A}-v_{B}\right)}{v_{B}} & \text { if } p_{B}^{B} \leq p_{B} \leq \breve{p}_{B}, \\ 0 & \text { if } p_{B} \geq \breve{p}_{B},\end{cases}
$$

and

$$
D_{A}= \begin{cases}\bar{\mu}-\frac{p_{A}-\alpha\left(v_{A}-v_{B}\right)}{v_{A}} & \text { if } p_{A} \leq \min \left\{\bar{p}_{A}, \breve{p}_{A}\right\} \\ \bar{\mu}-\left(\frac{p_{A}-p_{B}}{v_{A}-v_{B}}-2 \alpha\right) & \text { if } \breve{p}_{A} \leq p_{A} \leq p_{A}^{B}, \\ 0 & \text { if } p_{A} \geq p_{A}^{B}\end{cases}
$$

\section{Appendix II}

\section{Best reply functions in the price game}

\section{Firm $B$}

It follows from the demand functions that if $p_{A} \geq \bar{p}_{A}$, namely $\bar{\mu}-\mu_{A}<0$, then

$$
\Pi_{B}= \begin{cases}p_{B}\left(\bar{\mu}-\frac{p_{B}+\alpha\left(v_{A}-v_{B}\right)}{v_{B}}\right) & \text { if } p_{B} \leq \bar{p}_{B}, \\ 0 & \text { if } p_{B} \geq \bar{p}_{B} .\end{cases}
$$

From the first-order conditions (FOC), one can immediately write the best reply function $\varphi_{L}\left(p_{A}\right)$ :

$$
\varphi_{B}\left(p_{A}\right)= \begin{cases}\frac{1}{2}\left(\bar{\mu} v_{B}-\alpha\left(v_{A}-v_{B}\right)\right) & \text { if } \bar{p}_{B}>0, \\ {[0, y]} & \text { if } \bar{p}_{B} \leq 0 .\end{cases}
$$


In contrast, if $p_{A} \leq \bar{p}_{A}$, namely $\bar{\mu}-\mu_{A}>0$, then profit functions for firm $B$ turn out to be:

$$
\Pi_{B}= \begin{cases}p_{B}\left(\bar{\mu}-\frac{p_{B}+\alpha\left(v_{A}-v_{B}\right)}{v_{B}}\right) & \text { if } p_{B} \leq p_{B}^{B}, \\ p_{B}\left(\frac{p_{A}-p_{B}}{v_{A}-v_{B}}-2 \alpha-\frac{p_{B}+\alpha\left(v_{A}-v_{B}\right)}{v_{B}}\right) & \text { if } p_{B}^{B} \leq p_{B} \leq \hat{p}_{B}, \\ 0 & \text { if } p_{B} \geq \hat{p}_{B} .\end{cases}
$$

The FOC imply:

$$
p_{B}= \begin{cases}\frac{1}{2}\left(\bar{\mu} v_{B}-\alpha\left(v_{A}-v_{B}\right)\right) & \text { if } p_{B} \leq p_{B}^{B} . \\ \frac{1}{2 v_{A}}\left(p_{A} v_{B}-\left(v_{A}-v_{B}\right)\left(\alpha v_{A}+\alpha v_{B}\right)\right) & \text { if } p_{B}^{B} \leq p_{B} \leq \hat{p}_{B} .\end{cases}
$$

If $\bar{p}_{B} \leq 0$, then $\hat{p}_{B}<\tilde{p}_{B}<0$. Thus, firm $B$ 's profit is equal to 0 for all $p_{B} \in[0, y]$ and

$\varphi_{B}\left(p_{A}\right)=[0, y], \quad \forall p_{A}$.

If $\bar{p}_{B}>0$, then:

- if $\tilde{p}_{B} \leq p_{B}^{B}$, then $\varphi_{B}\left(p_{A}\right)=\tilde{p}_{B}$;

- if $\hat{p}_{B} \geq \max \left\{0, p_{B}^{B}\right\}$, then $\varphi_{B}\left(p_{A}\right)=\hat{p}_{B}$;

- if $0 \leq \hat{p}_{B} \leq p_{B}^{B} \leq \tilde{p}_{B}$, then $\varphi_{B}\left(p_{A}\right)=p_{B}^{B}$;

- if $\hat{p}_{B} \leq 0$, then the profit is equal to 0 and $\varphi_{B}\left(p_{A}\right)=[0, y]$.

Notice that the SOC are always satisfied.

\section{Firm $A$}

Applying the same rationale as above, one can derive that if $p_{B} \geq \bar{p}_{B}$, then

$$
\Pi_{H}= \begin{cases}p_{A}\left(\bar{\mu}-\frac{p_{A}-\alpha\left(v_{A}-v_{B}\right)}{v_{A}}\right) & \text { if } p_{A}<\bar{p}_{A}, \\ 0 & \text { if } p_{A} \geq \bar{p}_{A} .\end{cases}
$$

From the FOC, the best reply function is:

$$
\varphi_{A}\left(p_{B}\right)=\frac{1}{2} v_{A}\left(\bar{\mu}+\alpha \frac{1}{v_{A}}\left(v_{A}-v_{B}\right)\right) \quad \text { if } \bar{p}_{A}>0 .
$$

It is easy to check that the SOC are satisfied.

Otherwise, if $p_{B} \leq \bar{p}_{B}$, then the profit function is given by

$$
\Pi_{H}= \begin{cases}p_{A}\left(\bar{\mu}-\frac{p_{A}-\alpha\left(v_{A}-v_{B}\right)}{v_{A}}\right) & \text { if } p_{A} \leq \breve{p}_{A}, \\ p_{A}\left(\bar{\mu}-\left(\frac{p_{A}-p_{B}}{v_{A}-v_{B}}-2 \alpha\right)\right) & \text { if } \breve{p}_{A} \leq p_{A} \leq p_{A}^{B}, \\ 0 & \text { if } p_{A} \geq p_{A}^{B} .\end{cases}
$$

The FOC imply that:

$$
\tilde{p}_{A}= \begin{cases}\frac{1}{2} v_{A}\left(\bar{\mu}+\alpha \frac{1}{v_{A}}\left(v_{A}-v_{B}\right)\right) & \text { if } p_{A} \leq \breve{p}_{A}, \\ \left(\bar{\mu}+2 \alpha+\frac{p_{B}}{v_{A}-v_{B}}\right)\left(\frac{1}{2}\left(v_{A}-v_{B}\right)\right) & \text { if } \breve{p}_{A} \leq p_{A} \leq p_{A}^{B} .\end{cases}
$$

A simple computation suffices to that the SOC are satisfied. So,

- if $\tilde{p}_{A}<\breve{p}_{A}$, or $p_{B}>\frac{\left(\bar{\mu} v_{A} v_{B}-\left(v_{A}-v_{B}\right)\left(2 \alpha v_{A}+\alpha v_{B}\right)\right)}{2 v_{A}}$, then $\varphi_{A}\left(p_{B}\right)=\tilde{p}_{A}$;

- if $\breve{p}_{A} \leq \hat{p}_{A} \leq p_{A}^{B}$, or $p_{B}<\frac{\left(\bar{\mu} v_{B}-2 \alpha v_{A}-\alpha v_{B}+\alpha v_{B}\right)\left(v_{A}-v_{B}\right)}{\left(2 v_{A}-v_{B}\right)}$, then $\varphi_{A}\left(p_{B}\right)=\hat{p}_{A}$;

- if $\hat{p}_{A}<\breve{p}_{A}$, or

$$
\frac{\left(\bar{\mu} v_{B}-2 \alpha v_{A}-\alpha v_{B}+\alpha v_{B}\right)\left(v_{A}-v_{B}\right)}{\left(2 v_{A}-v_{B}\right)} \leq p_{B} \leq \frac{\left(\bar{\mu} v_{A} v_{B}-\left(v_{A}-v_{B}\right)\left(2 \alpha v_{A}+\alpha v_{B}\right)\right)}{2 v_{A}},
$$

then $\varphi_{A}\left(p_{B}\right)=\breve{p}_{A}$. 

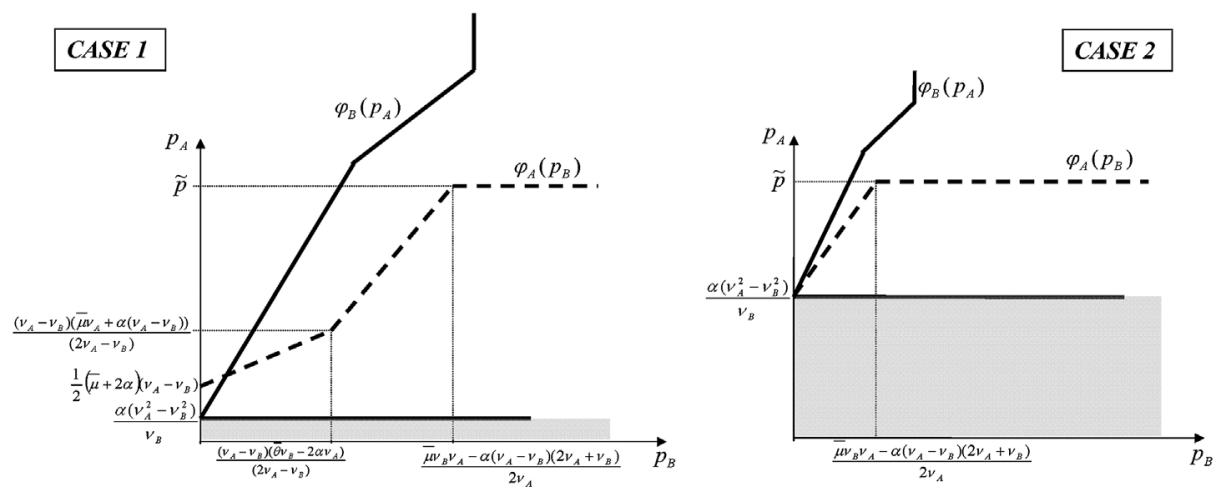

\section{CASE 3}
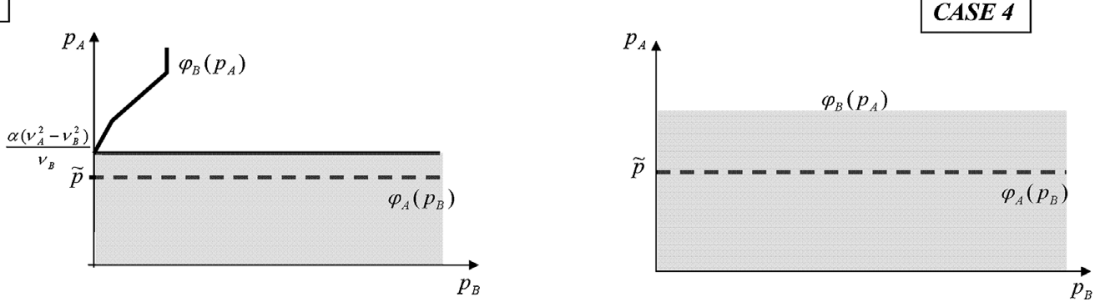

Figure 1 The intersection of best reply functions.

\section{Appendix III}

\section{Market structure at equilibrium}

Three cases have to be distinguished for the best reply function of firm $A$ at the second stage according to the sign of the thresholds of its definition domain, namely the sign of $\frac{\left(\bar{\mu} v_{A} v_{B}-\left(v_{A}-v_{B}\right)\left(2 \alpha v_{A}+\alpha v_{B}\right)\right)}{2 v_{A}}$ and $\frac{\left(\bar{\mu} v_{B}-2 \alpha v_{A}-\alpha v_{B}+\alpha v_{B}\right)\left(v_{A}-v_{B}\right)}{\left(2 v_{A}-v_{B}\right)}$.

To this end, notice that

$$
\frac{\left(\bar{\mu} v_{B}-2 \alpha v_{A}-\alpha v_{B}+\alpha v_{B}\right)\left(v_{A}-v_{B}\right)}{\left(2 v_{A}-v_{B}\right)}>0 \Leftrightarrow \frac{v_{A}}{v_{B}}<H_{1},
$$

with $H_{1}=\frac{\bar{\mu}}{/} 2 \alpha$, while

$$
\frac{\left(\bar{\mu} v_{A} v_{B}-\left(v_{A}-v_{B}\right)\left(2 \alpha v_{A}+\alpha v_{B}\right)\right)}{2 v_{A}}>0 \Leftrightarrow \frac{v_{A}}{v_{B}}<H_{2},
$$

with $H_{2}=\frac{1}{4 \alpha}\left(\alpha+\bar{\mu}+\sqrt{2 \alpha \bar{\mu}+9 \alpha^{2}+\bar{\mu}^{2}}\right)$. This implies that there are four cases of intersection between the best reply functions of both firms:

Case $1 \frac{v_{A}}{v_{B}}<H_{1}$;

Case $2 H_{1} \leq \frac{v_{A}}{v_{B}}<H_{2}$

Case $3 H_{2} \leq \frac{v_{A}}{v_{B}} \leq \frac{(\bar{\mu}+\alpha)}{\alpha}$;

Case $4 \frac{v_{A}}{v_{B}}>\frac{(\bar{\mu}+\alpha)}{\alpha}$.

These four cases are depicted in Figure 1. Notice from the figure that cases 3 and 4 can be combined because they lead to the same set of price equilibria. In case 1, firms $A$ and $B$ share the market, thereby both being active at some positive equilibrium prices; in the two remaining cases only firm $A$ can be active in the market at some positive equilibrium price. 


\section{Appendix IV}

\section{Proof of Proposition 4}

First, notice that the difference between the equilibrium $v_{A}-v_{B}$ is given by

$$
\frac{1}{7 \bar{\mu}-4 \alpha}\left(5 \bar{\mu}-\alpha-\sqrt{44 \alpha \bar{\mu}-23 \alpha^{2}+4 \bar{\mu}^{2}}\right) \bar{\nu} .
$$

In order to prove the statement in the Proposition 4, it suffices to show that $\frac{\partial}{\partial \alpha}\left(v_{A}-v_{B}\right)<0$. Indeed, given that $v_{A}=\bar{v}$, the difference $v_{A}(=\bar{v})-v_{B}$ can decrease if and only if $v_{B}$ increases. The derivative of the above expression with respect to $\alpha$, namely $\frac{\partial}{\partial \alpha}\left(v_{A}-v_{B}\right)$, boils down to

$$
\frac{73 \alpha-170 \bar{\mu}+13 \sqrt{44 \alpha \bar{\mu}-23 \alpha^{2}+4 \bar{\mu}^{2}}}{(7 \bar{\mu}-4 \alpha)^{2}\left(\sqrt{44 \alpha \bar{\mu}-23 \alpha^{2}+4 \bar{\mu}^{2}}\right)}(\bar{\mu}-\alpha) .
$$

While both terms in the denominator are positive, the term in the numerator is negative. So we can conclude that this derivative is negative, meaning that the higher the value of $\alpha$, the lower the value of $v_{A}-v_{B}$, or in other words (given $v_{A}=\bar{v}$ ), the higher the value of $\alpha$, the higher the value of $v_{B}$.

\section{References}

Conrad, K. (2005), "Price competition and product differentiation when consumers care for the environment," Environmental and Resource Economics 31, 1-19.

Copeland, B. R., and M. S. Taylor (1994), "North-South trade and the environment," Quarterly Journal of Economics 109, 755-87.

Elster J. (1989), “Social norms and economic theory,” Journal of Economic Perspectives 34, 99-117.

Gabszewicz, J. J., and J. F. Thisse (1979), “Price competition, quality and income disparities," Journal of Economic Theory 20, 340-59.

Gabszewicz, J. J., and J. F. Thisse (1980), “Entry (and exit) in a differentiated industry," Journal of Economic Theory 22, 327-38. Sanna-Randaccio, F., and R. Sestini (2012), "The impact of unilateral climate policy with endogenous plant location and market size asymmetry," Review of International Economics 20, 580-99. 